\title{
HOME AUTOMATION SYSTEM WITH WSN AND IOT
}

\author{
Dr Syeda Gauhar Fatima \\ Professor ECE dept, Deccan College of Engineering and Technology, \\ Darussalam, Hyderabad, India \\ Syeda Kausar Fatima
}

Associate Professor, Shadan College of Engineering and Technology, Hyderabad, India

Syed Mohd Ali

Research Scholar, ECE dept, JNTUH, Kukatpally, Hyderabad, India

Naseer Ahmed Khan

Student, ECE Dept, Deccan College of Engineering and Technology,

Darussalam, Hyderabad, India

Syed Adil

Student, ECE Dept, Deccan College of Engineering and Technology,

Darussalam, Hyderabad, India

\begin{abstract}
ABTRACT
Nowadays there is a brief usage and dependability of pulsating features of the smart devices. So there is a need of connecting these devices through internet to explore their functionality. In the world of Smart homes there exists different systems but failed to provide many functionalities like controlling the devices remotely, overhead in communication, and energy efficiency. This paper delivers the solution to the constraint of the existing systems. The monitoring and controlling of smart organized homes environmental and safety parameters a Wi-Fi based WSN system is designed. A lightweight MQTT protocol is used for interactions between devices and user. The user can flawlessly control and monitor the devices via Android Application using a Graphical User Interface (GUI) remotely.
\end{abstract}

Keywords: Wireless Sensor Network, Wireless Fidelity, MQTT, Android, Graphical User Interface, Home Automation System

Cite this Article: Dr Syeda Gauhar Fatima, Syeda Kausar Fatima, Syed Mohd Ali, Naseer Ahmed Khan and Syed Adil, Home Automation System with WSN and IOT, International Journal of Advanced Research in Engineering and Technology, 10(2), 2019, pp.78-85.

http://iaeme.com/Home/issue/IJARET?Volume $=10 \&$ Issue $=2$ 


\section{INTRODUCTION}

Home automation system represents the automatic sensation and electronic control towards household features, activities and appliances. It is an automation that replaces the electrical switch boards located in different rooms, and leads to ease and comfort life style for each member in home, especially the elders and physically handicapped members to operate them. Automation have a great impact after innovation, and open up as a serve many fields such as medical, industrial, corporation, building, transportation etc., and later on discovered and land to many homes, known as "Home automation" which includes controlling TV, Radio, DVDPlayer which can be establish in almost every home nowadays [1].Home automation system is utilization of information technologies and control system to reduce the human efforts. The fast growth of technologies affects us to use smart devices to remotely control the home appliances [3]. The paper shows the design and execution of a powerful, cost effective home automation system using Bluetooth technology. From many years the concept of home automation was limited to lighting and basic devices controlling, but recently the technology has been influence for the innovation of the organized world, allowing full control of our home from anywhere, to become reality. With home automation, you can rule how, when and why the devices should responds.

\subsection{Smart Homes with Internet of Things and WSN}

Internetworking of devices, automobiles and other electronic devices having sensing, intelligent abilities enable these devices to collect and barter data, giving an preface to Internet of Things. Nowadays most frequently use devices such as mobile, sensor, watch, TV are automated and connect to the Internet using a

wireless technology [1]. IoT makes them desirable of sharing, communicating and transporting data via Internet whether posting to a server or interpretation of data from a server. According to this paper, IoT is a technology which can easily connect home appliances to internet, can communicate to each other and provide a history for analytics which can understand human act, preferences etc. It is used to define such devices that have conceived from sensors and actuators. Low power, low data usage, etc. are hallmarks or signature of IoT devices [2]. Internet of Things is described as the environment in which the physical electronic devices with data sensing, processing capability which are used to communicate with other such device and process that allows data to take an smart decision which will prove an advantage for our daily day to day life style [7] .

IoT is a system of internetworking of devices that can be accessed via internet. Every new technology has vision towards many new challenges being opened up for those who are interested to adapt to the changes and IoT is one them. It's opening up with many challenges for together hardware state as well as software state and would start up for more standards in future years.

Smart Home system with the support of Internet of Things is the approach that all of our smart devices and home gadgets will be internetworked together to full control over all things of our home and more. In home automation, the working of devices is programmed and the rest of work is automated with personal bias.

Home Automation System notifies us about the events and actions that we want to know when we are not actually present at home. We can control the devices of house through our android devices anytime and anywhere as desired. 


\subsection{Home Automation using IoT and its}

Fig. 1: IoT Characteristics With advanced monitoring techniques of devices or objects by using sensors and connectivity among the devices, which would help them to make smarter decisions. The growth and coinciding of data, processes and things on the internet would make such connections more applicable, creating more opportunities for people, businesses and industries. With the help of interconnection of smart devices it is easy to collect more data from many places, fortifying various ways of increasing efficiency and improving safety and IoT security.

\subsection{Home Automation using Wireless Sensor Networks (WSN)}

Wireless sensor networks, are exposed to distributed self-directed sensors to supervise physical or environmental conditions such as temperature of environment, sound level, pressures and much more to co-operatively transfer this data through the network to other WSN network at other positions. A WSN can be technically stated as a network with wireless connectivity containing several distributed devices incorporated with sensors to monitor physical or environmental conditions.

In 2016, paper describes about the appetite of automation in wireless sensor networks brings up many revolutions in the existing technology due to its user friendly nature[4].The technologies of WSN are likely to be incorporated into the IoT, empowered for the global interconnection of different smart objects with advanced functionality. A wireless home automation network (WHAN) is primal enabling technology making intelligent and context aware indoor environments [8]. The user can practice smooth control over the devices in a smart home via the Android application based Graphical User Interface (GUI) on a Smart phone.

\subsection{Existing System}

The paper [3] represents the design and application of a powerful, low cost and user-friendly home automation system using Bluetooth technology. They have used Arduino board, Bluetooth module, sensors and smart phone application. This paper has remotely controlled the as well as also monitors the sensors. This system enables elderly and partially disabled people providing them a general-purpose home automation system, which can be easily applied in living home. They have used ultrasonic sensor for detection of water level and soil moisture detection sensor for automatic irrigation system which provides more relieve and facilities to users. HC-06 Bluetooth module has been interfaced along with fans and other devices with Arduino via relay. Smartphone application is used for serial communication between Smartphone and Bluetooth module which is further connected with Arduino board.

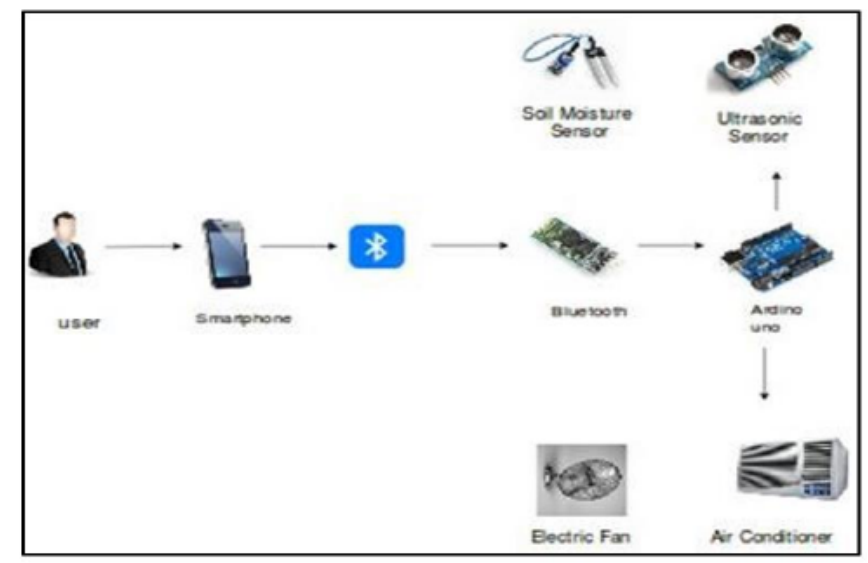

Figure 1 Existing System 


\section{PROPOSED SYSTEM}

This paper describes the method to facilitate an enhanced Home Automation System (HAS) using Android application for controlling different home appliances and monitoring them from anywhere, anytime. This emerge the concept of internetworking of smart devices with incorporation of Internet of things [IOT] and Wireless sensor network (WSN). This paper illustrate enhanced home automation that replaces the electrical switch boards located in different rooms, and leads to ease and comfort life style for each members in home, especially the elders and physically handicapped members to operate them. It provides information about the home appliances from monitoring anywhere, anytime using IOT. The system involves the use of a Wi-Fi module, Raspberry Pi, sensors and relays. The data received at the Gateway is sent through the UART serial port to the Wi-Fi module. The Wi-Fi module sends data to the server where it is stored and can be retrieved for future use. The user is provided with an android application based Graphic User Interface (GUI) to exercise the desired control over the lights, fan speed regulation, control of appliances, and information regarding the temperature, humidity, water tank level or rain. The additional benefits of this methodology are its security features which include fire alarms, burglar detection, and gas leakage detection. Mobile commands will be published to the Message Queuing Telemetry Transport (MQTT) server which is accessed by the user on the Smartphone via the android application.

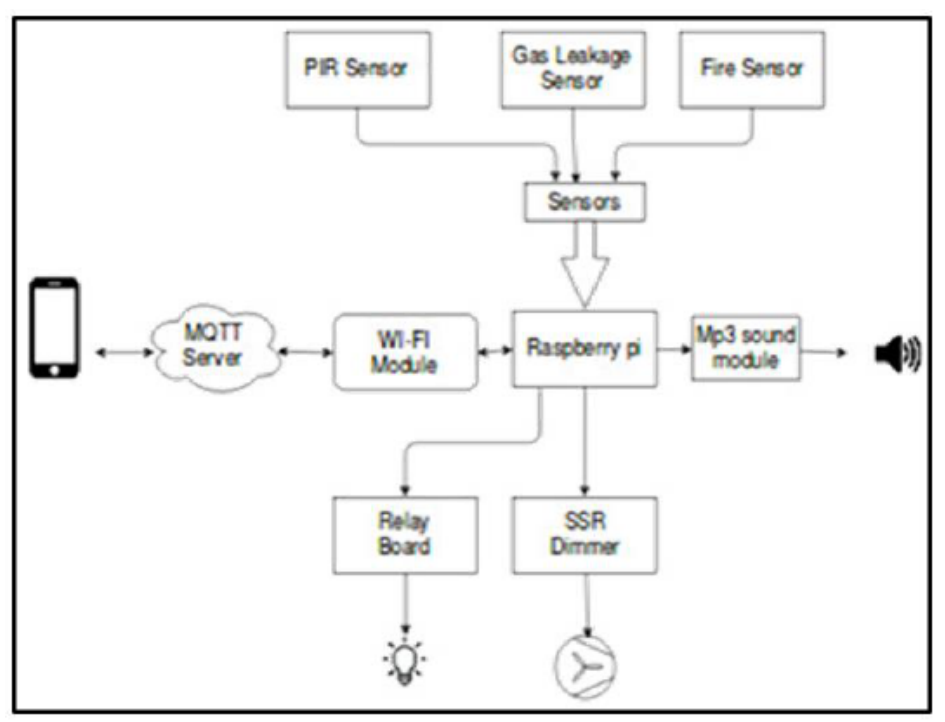

Figure 2 Proposed System

\subsection{Architecture}

The proposed system has two main architecture hardware and software architecture. The hardware architecture entails of raspberry pi, fire sensor, gas leakage sensor, PIR sensor, voice recognition module. The software architecture consists of IDE a development environment for raspberry pi and application which acts as a communicator between the software and the hardware. 1) Hardware architecture:

\subsubsection{Raspberry pi}

Raspberry Pi 3 is the latest generation of Raspberry Pi i.e. 3rd generation. It is credit card sized powerful computer which can be used for many applications.

Raspberry Pi 3 specifications are, it has Broadcom

BCM2387 chipset with $1.2 \mathrm{GHz}$ Quad-Core ARM CortexA53.The main feature of Raspberry Pi 3 is it has built-in Wi-Fi and Bluetooth. It has GPU and 1GB SDRAM. 
It has 40 GPIO pins through which different types of sensors can be interfaced.

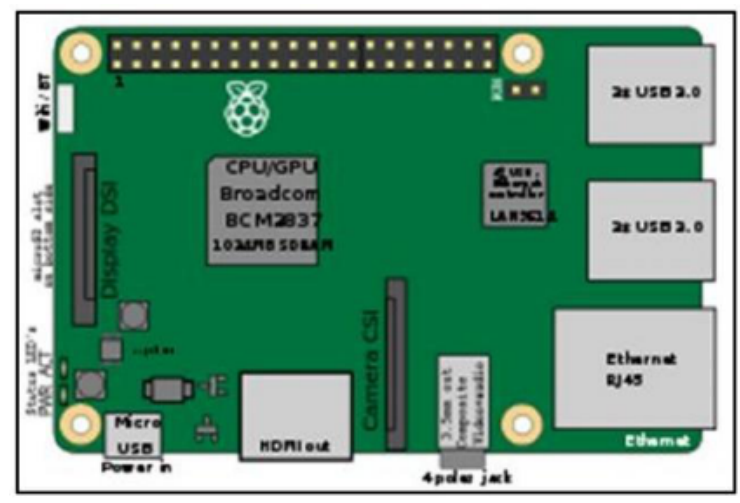

Figure 3 Raspberry Pi3

\subsubsection{Fire Sensor}

In the proposed system a fire sensor is used to respond to the presence of fire or flames. The fire sensor turns on alarm as soon as it detects fire and alerts about fire. In infrared flame detector, it uses dual IR of threshold ranges. Sensing the carbon dioxide emission is suitable for hydrocarbon fuels.

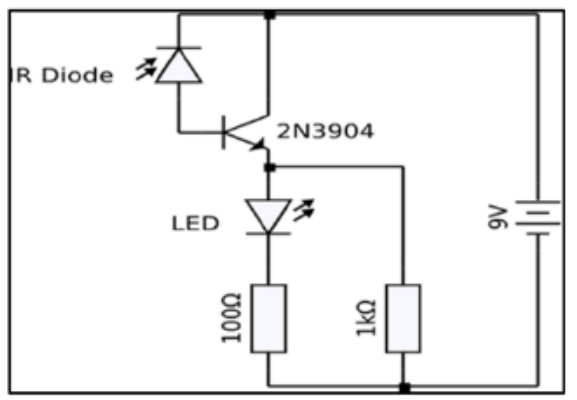

Figure 4 IR Fire Sensor

\subsubsection{Gas Leakage Sensor}

The gas leakage sensor senses the seepage of LPG gas. The gas leakage sensors in HAS is important as it can avoid the risk of gas leakage, if there exist leakage it turns on the alarm and alerts. With the help of SEN-1327 gas sensor module from RhydoLABZ the gas leakage is detected. The threshold is set using preset in module. Interfacing is done via SIP header. The sensor can also sense combustible gases like methane.

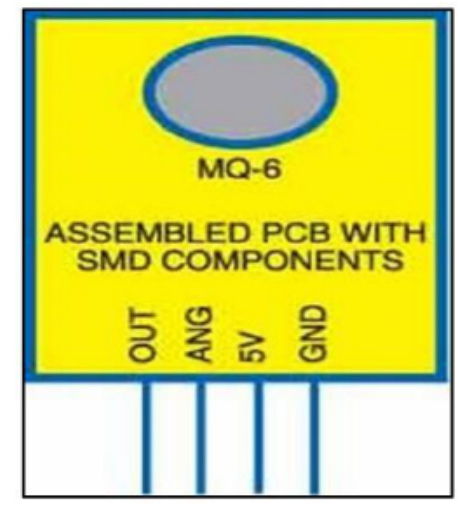

Figure 5 MQ-6 Gas Leakage 


\subsubsection{PIR Sensor}

PIR sensor are used to reflect infrared from the object in its field of range.PIR sensor is used as motion detector signal which can be used for burglar alarm, if any motion is detected then the buzzer sets on. The PIR sensor is placed at a place than increases its range i.e. at a place in house which covers the whole area.

\subsection{Software Architecture}

\subsubsection{AdaFruit WebIDE}

Adafruit WebIDE provides an interface for Raspberry Pi to perform programming functions, and allows compiling the source code of various languages like Python, Ruby, JavaScript and many others. AdaFruit can be accessed via Web browser on ports 8080 or 80 . It supports the easy compiling and running of source code packed with a debugger and visualiser for proper tracking, navigation of code and to test source code.

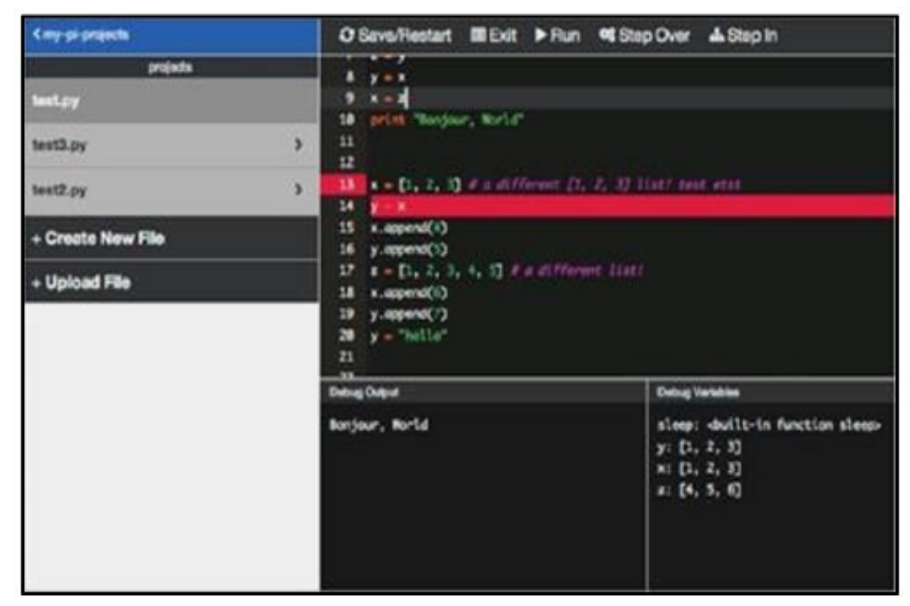

Figure 6 Adafruit WebIDE

\subsubsection{Software}

In software architecture application works as mediator between hardware and software .The hardware components can be controlled with the help of application. Application provides an interface for the user which makes it easy to use. Application provides graphical user interface (GUI) which increases the controlling efficiency. With the help of application home appliances can be controlled over a distance without any disturbance or difficulty. The application has certain switches that can be used for controlling the hardware.
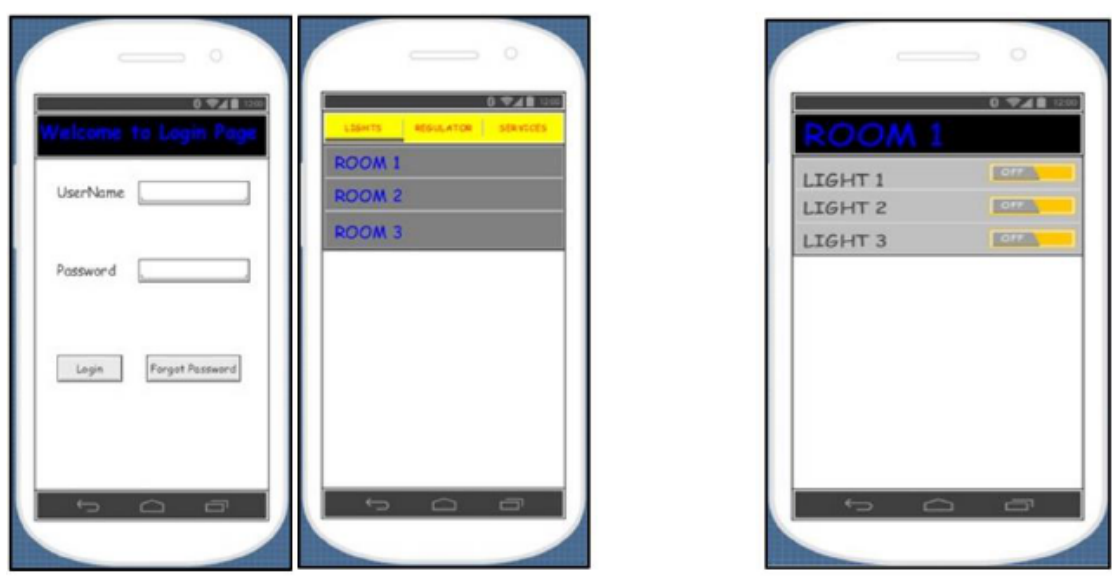

Figure 7 Android Application

Figure 8 Android Application Switches 


\section{APPLICATIONS}

An introduction to the technology of home automation system or smart homes can be described inside the home environment to provide comfort, amenity, security, ease and efficient use of energy to its inhabitant. Thus adding intellect to the home environment can provide a good quality of life. With the initiation of Internet of Things (IoT), the investigation and application of home automation are getting more preferred. Nowadays many scientists have done that provide home automation solution with the help of IoT.

\subsection{Lights and Fans}

The handler can examine the status of lights and fans through android device. The user can also switch on and off both lights as well as fans through the device without having bodily interaction with the switch board. This system is very beneficial for those users who cannot even reach up to the switches, thus providing limited movement.

\subsection{Gas Sensor}

The MQ-6 Gas Sensor module detects gas leakage in home. The MQ series of gas sensors use a small heater inside with an electrochemical sensor. They are excitable to a range of gasses and are used at room temperature. With its fast response time and high sensitivity, measurements are taken as soon as event occurred.

\subsection{Security System}

The security system can be implemented with the help of PIR (passive infrared sensor).This is based on PIR sensor with an IC that produces siren. The PIR sensor senses the infrared radiations emitted from the humans and it produces a digital output. This digital output is applied to the siren IC. Thus a buzzer rings when a human is sensed.

\section{CONCLUSION}

In this paper the aim is on developing Home Automation System with the backbone of IoT and WSN for monitoring and controlling the home electronic devices by using the flexible HAS Android Application. A varied range of sensors are used to capture the readings of temperature, gas and flame sensing devices forming it an effective system for security, controlling and monitoring.

With the specification of the system and simplicity of implementation leads for large scale development. This system can be very helpful for old aged homes, senior citizens and physically challenged people.

\section{FUTURE SCOPE}

Further this system can be developed by adding the topographies like controlling speed of fan and intensity of light through human brain or through blinking of eyes or the human meditation value and also some objects can be automated with the help of body or hand gestures.

\section{REFERENCES}

[1] "Worldwide Auto-mobi: Arduino IoT Home Automation System for IR Devices"- Ayad Ghany Ismaeel, Mohammed Qasim Kamal, International Conference on Current Research in Computer Science and Information Technology (ICCIT), Slemani - Iraq in 2017 
[2] "An Offline Online Strategy for IoT using MQTT"- Arya Sahadevan, Deepa Mathew, Jairam Mookatana, and Bijoy A. Jose, IEEE 4th International Conference on Cyber Security and Cloud Computing in 2017

[3] "Smart Home Automation System Using Bluetooth Technology"- Muhammad Asadullah, IEEE Student Member, Khalil Ullah, IEEE Member Department of Electrical Engineering National University of Computer and Emerging Sciences Peshawar, Pakistan in 2017.

[4] "An IoT based Home Automation Using Android Application"- P.Siva Nagendra Reddy, K.Tharun Kumar Reddy, P.Ajay Kumar Reddy, Dr.G.N.Kodanda Ramaiah,S.Nanda Kishor in International conference on Signal Processing, Communication, Power and Embedded System(SCOPES)-2016.

[5] "A Low Cost Home Automation System Using Wi-Fi Based Wireless Sensor Network Incorporating Internet of Things (IoT)" - Vikram.N, Harish K.S, Nihaal M.S,Raksha Umesh, Shetty Aashik Ashok Kumar in IEEE year 2017

[6] "Development of Remotely Controlled Home Automation System"- K.H. Mukendi, M. Adonis in IEEE 2017.

[7] "An enhanced security framework for home appliances in smart home"- Won Min Kang, Sea Moon and Jong Hyuk Park, year 2017.

[8] "Home Automation Using Internet of Things"- Shewta Singh, Kishore Kumar Ray, in International Journal of Computer Engineer and Applications. Pp. 1-9, Www.ijcea.com, ISSN 2321-3469.

[9] "Wireless home automation networks for indoor surveillance:technologoies and experiments" - milo Spadacini,Stefano Savazzi and Monica Nicoli, in EURASIP Journal 2014 http://jwcn.eurasipjournals.com/content/2014/1/6.

[10] https://www.raspberrypi.org

[11] https://www.engineersgarage.com

[12] https://en.m.wikipedia.org/wiki/Passive_infrared_sensor

[13] https://en.m.wikipedia.org/wiki/Flame_detector

[14] https://github.com/mqtt/mqtt.github.io/wiki

[15] https://commons.wikimedia.org/wiki/File:Raspberry_Pi_B\%2B_rev_1.2.svg

[16] http://electronicsforu.com/electronicsprojects/hardware-diy/gas-leakage-alarm

[17] Deepika Yadav, Yogendra Singh and Harshit Gupta, Controlling of Relay using Raspberry Pi Via Internet for Home Automation. International Journal of Advanced Research in Engineering and Technology, 9(1), 2018, pp 1-11

[18] T. Sai Pranay, G. Durga Bhavani, P. SivaSravani, Dr Sudhir Ranjan Pattanaik, Intelligent Voice Controlled Home Automation System, International Journal of Mechanical Engineering and Technology 9(1), 2018, pp. 640-645.

[19] M. Shri Harish, Syed Riyaz Ahammed, R.Hushien, Manoj Kumar.V, Bachina Harish Babu, Optimized Water Pumping System Using Arduino for Home Automation, International Journal of Mechanical Engineering and Technology 8(8), 2017, pp. 1412-1416. 